\title{
Art on a Plate: A Pilot Evaluation of an International Initiative Designed to Promote Consumption of Fruits and Vegetables by Children
}

\author{
Klazine van der Horst, $\mathrm{PhD}^{1,2}$; Kevin C. Mathias, $\mathrm{PhD}^{1}$; Alberto Prieto Patron, $\mathrm{PhD}^{1}$; \\ Xavier Allirot, $\mathrm{PhD}^{3}$
}

\begin{abstract}
Objective: To evaluate the 2016 International Chefs Day cooking workshops Art on a Plate.

Design: Nonexperimental pretest-posttest design

Setting: Art on a Plate workshops with children from 14 countries in Asia, America, and Europe.

Participants: A total of 433 workshop participants aged 4-14 years (mean age, 8.6 years).

Intervention: Instructed by a chef, children in the workshops created a self-chosen design on their plate with a spinach-fruit salad.

Main Outcome Measures: Before and after the workshop, a questionnaire assessing liking and willingness to eat or taste; hunger was assessed using the Teddy the Bear method and emotions were assessed using the Self-assessment Manikin. The event coordinator evaluated salad intake.

Analysis: Linear and generalized linear (logit) mixed models were used to test statistical differences before and after the workshop.

Results: The workshop resulted in a small increase in liking $(n=409 ; P=.02)$ and person control $(\mathrm{n}=375 ; \mathrm{P}<.001)$ and a decrease in hunger $(\mathrm{n}=379 ; \mathrm{P}<.001)$. A total of $30 \%$ of children increased their liking scores, $18 \%$ decreased them, and $52 \%$ did not change them. Significant associations of liking and change in liking with salad intake were in the expected direction.

Conclusion and Implications: This study showed the positive effect of a cooking workshop on children's salad liking across a selection of countries worldwide. Further research and novel methods are needed to evaluate the long-term effectiveness of cooking activities in real-life settings across countries.
\end{abstract}

Key Words: acceptance, children, cooking, meal preparation, vegetable intake (J Nutr Educ Behav. 2019; 51:919-925.)

Accepted March 20, 2019. Published online April 30, 2019.

\section{INTRODUCTION}

Although the promotion of healthy eating habits in children is considered to be highly important, school-based interventions, which often focus on increasing fruit and vegetable intake, have had minimal impact on vegetable intake. $^{1,2}$ Simultaneously, vegetable intake remains a parental concern. ${ }^{3-5}$ The use of experiential learning strategies in school education programs is associated with the largest increases in fruit and vegetable consumption, preference, and nutritional knowledge outcomes. $^{6-8}$ The mechanism behind the

\footnotetext{
${ }^{1}$ Nestec Ltd, Nestlé Research Center, Institute of Nutritional Science, Vers-Chez-Les-Blanc, Lausanne, Switzerland

${ }^{2}$ Department of Health Professions, Bern University of Applied Sciences, Bern, Switzerland

${ }^{3}$ Basque Culinary Center, San Sebastián, Spain

Conflict of Interest Disclosure: The authors' conflict of interest disclosures can be found online with this article on www.jneb.org.

Address for correspondence: Klazine van der Horst, PhD, Department of Health Professions, Bern University of Applied Sciences, Murtenstrasse 10, CH-3008 Bern, Switzerland; E-mail: Klazine.vanderhorst@bfh.ch

(C) 2019 The Authors. Published by Elsevier Inc. on behalf of Society for Nutrition Education and Behavior. This is an open access article under the CC BY-NC-ND license. (http:// creativecommons.org/licenses/by-nc-nd/4.0/)

https://doi.org/10.1016/j.jneb.2019.03.009
}

positive effects of participatory activities (eg, cooking foods) potentially relates to several factors, such as having choices, ${ }^{9,10}$ increasing familiarity with foods, ${ }^{11,12}$ a higher degree of child control and a consequent level of intrinsic motivation and pride, ${ }^{10,13,14}$ the higher value placed on self-created objects, $^{15-17}$ and the positive context in which cooking activities take place. $^{18,19}$

In addition to intervention studies that often combined several elements such as education and gardening activities with cooking, 2 experimental studies assessed the immediate effect of cooking on subsequent intake. Results from these experimental studies showed increased vegetable intake $^{20}$ and increased willingness to choose and taste unfamiliar foods with vegetables. ${ }^{21}$ Observational studies showed that cooking activities were associated with diet quality and 
fruit and vegetable intake in children $^{22,23}$ and adolescents. ${ }^{24}$

In addition to participatory activities, several factors such as role modeling, ${ }^{25}$ availability (ie, whether the food is present), ${ }^{25,26}$ accessibility (eg, washed and cut into pieces), ${ }^{27}$ and enhancing the visual appeal of foods were suggested as strategies for promoting fruit and vegetable intake in children. ${ }^{28,29}$

An international cooking workshop was developed with the aim of creating a positive hands-on experience with fruits and vegetables for children. The workshops were organized during the International Chefs Day on October 20, 2016. International Chefs Day is celebrated by over 10 million chefs around the world. It is an event for chefs to engage with their communities, honor their profession, and share the pleasure of cooking. Nestlé Professional and the Nestlé Healthy Kids Program ${ }^{30}$ initiated a yearly event in 2015 with cooking workshops for children together with professional chefs in over 60 countries. In 2016, the workshops were conducted in 62 countries, with approximately 2,000 chefs and 19,000 children participating in the cooking workshops.
The workshops in 2016 were themed Art on a Plate and combined hands-on experience with other important determinants of fruit and vegetable intake, such as exposure, role modeling, and visual appeal. The objective of the current study was to assess the effectiveness of the workshops in achieving positive change in children's liking and willingness to consume the prepared fruits and vegetables. It was hypothesized that children's liking and willingness to consume the prepared fruits and vegetables would increase after completing the workshop.

\section{METHODS}

\section{Participants and Recruitment}

The 52 Nestlé Healthy Kids contact persons from countries that participated in the Art on a Plate event were asked to participate in the evaluation on a voluntary basis as well, which resulted in 17 countries contributing to the evaluation. All countries organized cooking workshops in 1 location, except Russia, where the 3 workshops were organized in different locations. Each participating location conducted at least 1 workshop. The
Healthy Kids contact persons invited children to participate most often through collaborations with schools. Parents provided written consent for a child to participate in the event and evaluation. The study was exempt from ethical approval because it did not include health measures or sensitive or harmful questions and data were collected anonymously. ${ }^{31}$ Before the analyses were conducted, 3 countries were excluded from the study because the study protocol was not followed adequately, only part of the data was collected, or the pre- and post-questionnaires were not assigned a unique identifier and therefore could not be matched to the corresponding child. In total, 433 children from 14 countries and 16 different locations were included in the study. Table 1 lists the descriptive statistics of the participating countries and the total study sample.

\section{Study Protocol}

Countries participating in the evaluation were free to set up the Art on a Plate workshop content with the chefs following local wishes and needs. However, to have comparable workshops across the evaluating

\begin{tabular}{|c|c|c|c|c|c|c|c|c|c|c|}
\hline \multirow[b]{2}{*}{ Country } & \multirow[b]{2}{*}{$\mathbf{n}$} & \multirow[b]{2}{*}{ Girls (\%) } & \multirow[b]{2}{*}{$\begin{array}{c}\text { Age, } \\
\text { y (mean) }\end{array}$} & \multirow[b]{2}{*}{$\begin{array}{c}\text { Age, } \\
\text { y (range) }\end{array}$} & \multicolumn{3}{|c|}{ Spinach Intake } & \multicolumn{3}{|c|}{ Other Ingredient Intake } \\
\hline & & & & & $\begin{array}{c}\text { None } \\
(\%)\end{array}$ & $\begin{array}{c}\text { Taste } \\
(\%)\end{array}$ & $\begin{array}{l}\text { Eat } \\
(\%)\end{array}$ & $\begin{array}{c}\text { None } \\
(\%)\end{array}$ & $\begin{array}{c}\text { Taste } \\
(\%)\end{array}$ & $\begin{array}{l}\text { Eat } \\
\text { (\%) }\end{array}$ \\
\hline Argentina & 48 & 50 & 9.4 & $9-11$ & 40 & 33 & 27 & 0 & 20 & 80 \\
\hline Austria & 8 & 50 & 6.5 & $4-8$ & 0 & 0 & 100 & 0 & 0 & 100 \\
\hline Brazil & 17 & 41 & 7.7 & $7-8$ & 24 & 47 & 29 & 18 & 47 & 35 \\
\hline China $^{a}$ & 41 & 65 & 8.2 & $7-10$ & 0 & 0 & 100 & 0 & 0 & 100 \\
\hline India & 12 & 50 & 8.6 & $6-14$ & 8 & 33 & 58 & 8 & 42 & 50 \\
\hline Indonesia & 29 & 59 & 10.6 & $9-12$ & 52 & 48 & 0 & 14 & 86 & 0 \\
\hline México & 23 & 65 & 7.7 & $7-8$ & 5 & 32 & 64 & 0 & 23 & 77 \\
\hline Peru & 15 & 47 & 5.0 & $5-5$ & 7 & 40 & 53 & 0 & 33 & 67 \\
\hline Philippines & 51 & 53 & 9.3 & $9-14$ & 16 & 37 & 47 & 0 & 4 & 96 \\
\hline Poland & 10 & 50 & 9.2 & $8-11$ & 0 & 60 & 40 & 0 & 40 & 60 \\
\hline Portugal & 46 & 43 & 6.8 & $4-9$ & 24 & 27 & 48 & 18 & 15 & 67 \\
\hline Slovak Republic & 22 & 64 & 7.4 & $7-8$ & 14 & 27 & 59 & 0 & 0 & 100 \\
\hline Switzerland & 55 & 62 & 9.0 & $6-12$ & 11 & 47 & 42 & 8 & 26 & 66 \\
\hline Bryansk, Russia & 13 & 69 & 9.5 & $9-10$ & 77 & 0 & 23 & 0 & 0 & 100 \\
\hline Kineshma, Russia & 22 & 41 & 7.9 & $7-8$ & 0 & 32 & 68 & 0 & 18 & 82 \\
\hline Moscow, Russia & 21 & 67 & 8.8 & $7-9$ & 0 & 48 & 52 & 0 & 43 & 57 \\
\hline Total & 433 & 55 & 8.4 & $4-14$ & 18 & 33 & 49 & 4 & 23 & 73 \\
\hline
\end{tabular}

${ }^{a}$ China reported that children were asked to consume the salad to prevent food waste.

Note: Instructed by a chef, children in the workshops created a self-chosen design on their plate with a spinach-fruit salad. All countries organized a cooking workshop in 1 location, except Russia, in which 3 workshops were organized in different locations. 
countries, the contact persons were provided with a study protocol and were asked to include 1 specific recipe for spinach and fruit salad in the workshop. Contact persons were briefed through a telephone conference regarding how to use the evaluation protocol and measurement instruments. Questionnaires were translated into the local language in the respective countries. Children participated in groups in a single cooking workshop of about 1 hour with chefs in which they created an attractive design with the ingredients based on a spinach and fruit salad recipe. Spinach was used as an ingredient for the salad in all of the countries. The other ingredients could remain unchanged or be adapted depending on the availability of each ingredient, local taste, and the ideas of the chefs. The children were asked to use spinach for the Art on a Plate design but were free to choose from other ingredients that were provided. After the workshop, the children had the opportunity to taste and eat the salad they created. In some countries, children could make a second recipe during the workshop; however, this was not part of the evaluation.

\section{Measurements}

A questionnaire was administered before and after the workshop and answered by the children. If needed, children were assisted by the local organization members. Willingness to taste and eat was assessed with the questions If for your next meal you were offered a Spinach and Fruit Salad, would you like to taste it? and Do you think you would eat it entirely? Both questions were measured on a yes/no answering scale. This was followed by a third question assessing liking of the entire meal/salad, How much would you like it? on a 5-point smiley scale. The Self-Assessment Manikin (SAM) was used to assess children's emotions. $^{32}$ The SAM is a validated nonverbal pictorial assessment technique that directly measures the valence (pleasure), arousal, and dominance (control) associated with a person's affective reaction to a stimulus and is an easy method for quickly assessing reports of affective response in various contexts, including cooking. ${ }^{20}$ Children were asked to rate their current state on each of the 3 dimensions on a 9-point pictorial scale. The pleasure dimension ranged from a smiling happy figure to a frowning unhappy figure. The arousal dimension ranged from an excited wide-eyed figure to a relaxed sleepy figure. The dominance dimension represented changes in control with changes in the size of the SAM; a large figure indicated maximum control of the situation. ${ }^{32}$ Hunger and fullness were assessed with Teddy the Bear ${ }^{33}$ on a 5-point verbal pictorial scale that indicated the amount of food in the belly of a teddy bear; verbal indications ranged from I am really hungry! My belly feels very empty and is rumbling! to I am not hungry at all! My belly feels very full and I cannot eat any more food! The event organizers were asked to evaluate the salad intake based on 2 questions. The first question asked whether the child ate the spinach on the plate, according to the following answers: the child did not eat and taste the spinach, the child tasted only, or the child ate the spinach. The second question asked whether the child ate the other ingredients from the plate; the answer options were: the child did not eat and taste the other ingredients, the child tasted only, or the child ate the other ingredients.

\section{Statistical analysis}

The researchers conducted statistical analyses using Stata software (version 13.1, StataCorp LP, College Station, TX, 2013). Because there were only 2 measurements (pre and post workshop), all analyses included subjects who reported both pre- and postmeasures. Linear and generalized linear (logit) mixed models were used to test statistical differences between before and after the workshop for willingness to taste and eat entirely, liking, emotions, and hunger. Models included a coefficient for time (post- minus pre-workshop), a fixed effect for gender, a random intercept for each subject (level 1), and a random intercept for each workshop location (level 2). Age was not included in the models owing to the high correlation with the workshop location. To examine whether the effect of the intervention on liking differed by initial liking, additional coefficients for initial liking (indicator variable) and the interaction between initial liking and time were included in the model. The overall interaction was significant; therefore, the change in liking across each level of initial liking was estimated using the post-estimation -lincom command and tested whether it was statistically different from 0 . Mixed ordered logit models with coefficients for gender, initial liking (indicator variable), difference in liking (no difference, decrease, and increase), and a random intercept for each workshop location were used to examine the association of difference in liking after the workshop and intake of the salad reported as did not taste, tasted, and ate. Subjects from China were excluded from this particular analysis because they were encouraged to prevent food waste and eat all of the food. The researchers checked the proportional odds assumption for the ordered logit models using a Brant test without the random intercepts. In addition, the proportional odds assumption was checked using the model including the random intercepts by collapsing the intake variables into the logistic models of tasted and ate vs did not taste, and ate vs did not taste and tasted, and comparing the coefficients from the 2 logistic models. Associations of emotions and liking scores with intake were tested using models similar to those reported previously.

\section{RESULTS}

In total, data from 433 children (55\% of whom were girls) were included in the cooking workshop evaluation. Mean age of children was 8.4 years, but with a large variation ranging from 4 to 14 years (Table 1). The size of the event in the number of participants differed among countries; the smallest number of children was in Austria, and the largest in Switzerland.

In total, $18 \%$ of children did not eat or taste the spinach, 33\% tasted only, and $49 \%$ ate the spinach. The other ingredients (mainly fruit) were not eaten by $4 \%$ of the children, 


\begin{tabular}{lccccc} 
Outcomes & $\mathbf{n}^{\mathbf{a}}$ & $\begin{array}{c}\text { Initial } \\
\text { Response }\end{array}$ & $\begin{array}{c}\text { Post-Workshop } \\
\text { Response }\end{array}$ & $\begin{array}{c}\text { Odds Ratio } \\
\text { (95\% Confidence } \\
\text { Interval) or } \\
\text { Difference } \pm \text { SE }\end{array}$ & $\boldsymbol{P}$ \\
Would you like to taste it (\% yes) & 410 & 89.5 & 90.1 & $1.08(0.70-1.67)$ & .74 \\
Would you like to eat it entirely (\% yes) & 403 & 42.3 & 47.1 & $1.27(0.91-1.78)$ & .15 \\
Liking (1-5) (mean) & 409 & 4.06 & 4.20 & $0.14 \pm 0.06$ & .02 \\
Valence (pleasure) (1-9) (mean) & 385 & 8.31 & 8.18 & $-0.13 \pm 0.07$ & .07 \\
Arousal (1-9) (mean) & 375 & 5.85 & 5.90 & $0.15 \pm 0.14$ & .73 \\
Dominance (control) (1-9) (mean) & 375 & 6.77 & 7.16 & $0.39 \pm 0.11$ & $<.001$ \\
Hunger (1-5) (mean) & 379 & 3.31 & 2.49 & $-0.82 \pm 0.08$ & $<.001$ \\
\hline
\end{tabular}

${ }^{a} n$ represents the sample with complete data for a measure before and after the workshop.

Notes: Instructed by a chef, children in the workshops created a self-chosen design on their plate with a spinach-fruit salad. Differences between initial and post-workshop responses were estimated and tested for statistical significance using mixed models including a fixed effect for gender, a random intercept for each subject (level 1), and a random intercept for each workshop location (level 2).

whereas 23\% tasted only and 73\% ate the other ingredients (Table 1).

Overall the workshop showed a small but significant increase in the liking score $(\Delta=0.14 ; P=.02)$, the emotion dominance $(\Delta=0.39$; $P<.001)$, and a significant decrease in hunger $(\Delta=-0.82 ; \quad P<.001)$ (Table 2). Willingness to eat and taste and other emotions did not significantly differ before and after the workshop.

Comparison of values before and after the workshop showed that 30\% of children increased their liking score, whereas $18 \%$ of children decreased their liking scores and the remaining $52 \%$ did not change the scores (Table 3 ). The changes in liking scores were significantly associated with post-cooking workshop intake (Table 4).

The association between both increased and decreased liking scores and intake was in the expected direction compared with that for subjects who did not change their liking for the salad. A decrease in liking was significantly associated with a lower likelihood of being at a higher intake level of spinach (odds ratio $[\mathrm{OR}]=0.30 ; 95 \%$ confidence interval [CI], 0.16-0.56) and other ingredients $(\mathrm{OR}=0.36 ;$ 95\% CI, 0.18-0.72). An increase in liking was significantly associated with a higher likelihood of being in a higher intake category of other ingredients $(\mathrm{OR}=2.8 ; 95 \% \mathrm{CI}$,
1.3-6.0). Percentages of subjects by intake levels across change in liking were predicted at an initial liking of 3 to provide a context for the ORs from the ordered logit models in Table 4 (Figure).

Initial $(P=.001)$ and post $(P=.001)$ liking scores were both significant and positively associated with spinach intake. Initial and post scores for valence (pleasure) were not associated with higher intake levels, higher initial arousal was significantly associated with lower intake levels of spinach $(P=.04)$, and post-dominance (control) was significant and positively associated with post-liking scores $(P=.003)$ but not with intake (data not presented).

Table 3. Change in Liking After Workshop, by Initial Liking Score

\begin{tabular}{|c|c|c|c|c|c|c|}
\hline \multirow[b]{3}{*}{$\begin{array}{l}\text { Initial Liking } \\
\text { Score }\end{array}$} & & \multicolumn{3}{|c|}{ Change in Liking (Initial vs Post) } & \multirow[b]{3}{*}{ Change $^{b} \pm$ SE } & \multirow[b]{3}{*}{$P^{c}$} \\
\hline & & Decrease & Same & Increase & & \\
\hline & $\mathbf{n}$ & \multicolumn{3}{|c|}{$\%$ Within Each Level of Initial Liking ${ }^{a}$} & & \\
\hline 1 & 23 & 0 & 39 & 61 & $1.8 \pm 0.2$ & $<.001$ \\
\hline 2 & 13 & 8 & 15 & 77 & $1.7 \pm 0.3$ & $<.001$ \\
\hline 3 & 63 & 16 & 19 & 65 & $0.68 \pm 0.13$ & $<.001$ \\
\hline 4 & 129 & 23 & 33 & 44 & $0.06 \pm 0.09$ & .50 \\
\hline 5 & 181 & 18 & 82 & 0 & $-0.32 \pm 0.08$ & $<.001$ \\
\hline Total & 409 & 18 & 52 & 30 & & \\
\hline
\end{tabular}

${ }^{a}$ Descriptive percentages of children decreasing, remaining the same, and increasing their liking by initial liking score; ${ }^{\text {b }}$ redicted change in liking score using linear combinations of the model coefficients. The estimate of change is calculated as post liking minus initial liking; ${ }^{\mathrm{T}}$ Tests for whether change in liking score differed from 0.

Notes: Instructed by a chef, children in the workshops created a self-chosen design on their plate with a spinach-fruit salad. A mixed model with covariates for gender, time (pre vs post workshop), initial liking score, time interacted with initial liking, and random coefficients for study location were used to examine whether the effect of the intervention on liking differed by the initial liking score. 
Table 4. Associations of Change in Liking With Post-Workshop Intake of Spinach and Fruit Salad $(n=361)$

Spinach

\section{Variables}

Sex (girl)

Initial liking score

Dislike very much (1)

Dislike slightly (2)

Neither like nor dislike (3)

Like slightly (4)

Like very much (5)

Difference in liking

Same liking

Like less than initial

Like more than initial

\section{Odds Ratio (95\% \\ Confidence Interval)}

$1.1(0.71-1.7)$

Reference

$1.1(0.28-4.2)$

$2.1(0.75-5.6)$

$2.9(1.1-7.5)$

$\mathbf{5 . 7}(2.1-15.8)$

Reference

$\mathbf{0 . 3 0}(0.16-0.56)$

$1.7(0.92-3.1)$
Other Ingredients

\begin{tabular}{cr}
\hline Odds Ratio (95\% & \\
Confidence Interval) & $\boldsymbol{P}$ \\
$1.0(0.59-1.7)$ & .99 \\
& \\
Reference & \\
$0.2(0.051 .2)$ & .09 \\
$1.4(0.42-4.6)$ & .58 \\
$2.5(0.787 .7)$ & .12 \\
$\mathbf{4 . 1}(1.2-13.5)$ & .02
\end{tabular}

Reference

$<.001$

.09

$\mathbf{0 . 3 6}(0.18-0.72)$

.004

$2.8(1.3-6.0)$

Notes: Instructed by a chef, children in the workshops created a self-chosen design on their plate with a spinach-fruit salad. Mixed ordered logit models including gender as a fixed effect and workshop location as a random effect were conducted to estimate the association of difference in liking (pre vs post) with intake of spinach and other ingredients. Odds ratios are in favor of eating vs tasting/none or eating/tasting vs none. Subjects in the workshop in China were excluded from this particular analysis because they were asked to consume the salad to prevent food waste. Significant odds ratios are indicated in bold.
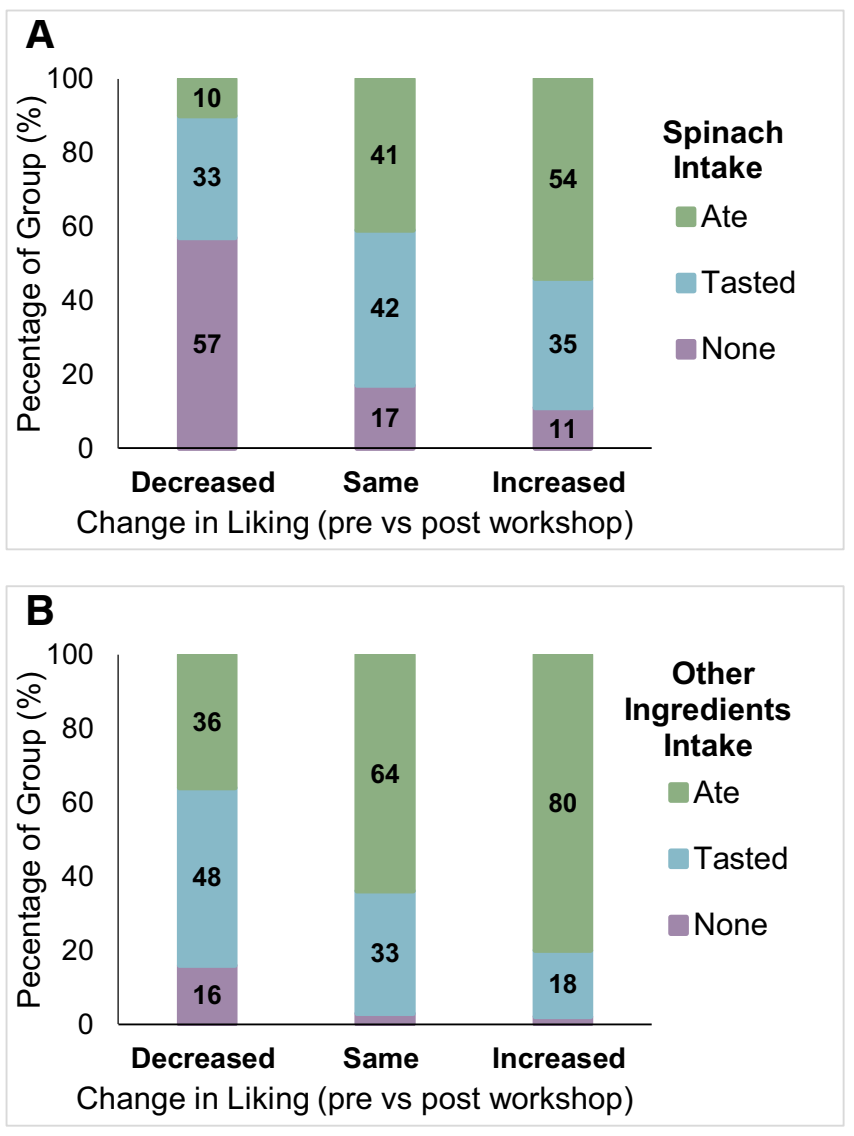

Figure. Post-workshop intake of spinach (A) and other ingredients (B) by change (pre vs post workshop) in liking scores $(n=361)$. Instructed by a chef, children in the workshops created a self-chosen design on their plate with a spinach-fruit salad. Percentages of change were predicted using coefficients from mixed ordered logit models with post-workshop intake categories as the outcome, change in liking as the primary exposure of interest, initial liking as a control variable (set at 3), gender as a covariate, and workshop location as a random effect.

\section{DISCUSSION}

Cooking workshops provide an opportunity to promote fruit and vegetable intake in children. Results of this evaluation study showed that most of the children tasted or ate the spinach and fruit salad. The results are in line with former research showing that children who participated in cooking a meal increased their intake of a subsequent lunch meal and increased their willingness to choose and taste unfamiliar foods with vegetables. ${ }^{20,21}$ The positive effects on liking may have resulted from various factors, 1 of which was the positive social context in which the workshops took place. ${ }^{34}$ Instead of a normal school day, children were participating in a cooking workshop with a real chef. In the development of food likes and dislikes, the context or atmosphere in which the food exposure takes place is mentioned as important. ${ }^{12}$ In cooking workshops, children are exposed to foods in a positive environment. Children reported that they enjoyed cooking and that cooking stimulated positive feelings such as ownership and pride. ${ }^{13,14}$ In line with a previous study, ${ }^{20}$ the children reported an increased feeling of dominance after the cooking workshop. However, a positive effect on the emotion valence (pleasure) could not be confirmed. This could be because of 
the setting of a school outing with an already high level of valence before the workshop started.

In addition to this positive environment, other factors might have contributed to the increase in liking of the salad. First, the pre-workshop questionnaire showed that the spinach and fruit salad were well-liked. Most children indicated a willingness to taste it and the sample of children had a high initial liking score on average. Although spinach is a vegetable that children often reject, the combination with fruits that are well-liked by children might add positive value to the dish. A former study showed that serving a moderately liked food mixed with a preferred one might support the acceptance of the moderately liked food. ${ }^{35}$ Other factors contributing to an increase in liking and likelihood of consumption were the enhanced visual appeal of foods through the Art on a Plate $\operatorname{design}^{28,29,36}$ and the role model function of a chef. Role modeling was mainly explored in the parent -child relationship and parental modeling was consistently shown to have significant and positive associations with children's fruit and vegetable intake. ${ }^{37,38}$ Because chefs are well-qualified to teach professional cooking skills and instill enthusiasm for cooking with fruits and vegetables, they can act as positive role models.

Owing to the setting of the workshops as a natural event, several limitations apply to this study, such as the absence of a control group, the lack of a baseline intake measurement of the salad without a workshop, and the absence of a precise intake measurement in grams. Another limitation was that liking and willingness to taste and eat were measured for the full salad; separate measures for the fruit and spinach were not collected. Therefore, children might have answered the questions more positively thinking about the fruits, or potentially more negatively if focusing only on the spinach. In addition, the questionnaire was not pretested in the various countries. This might have led to improper interpretation owing to cultural differences, especially in countries where these questions had never before been applied. Moreover, this study assessed the effectiveness of 1 short event and the potential sustainability of the effects could not be explored. Changes in liking or consumption might only have been in the short term. If repeated regularly at home, fun derived from the novelty might diminish over time. However, alternating between strategies to keep healthy foods interesting and children engaged might be successful if they lead to regular offering and tasting of the foods. It was shown that repeated exposure increases intake over time. $^{39,40}$

The variability in ingredients and organization in the different locations could be seen as a limitation because it prevented standardization and resulted in less controlled implementation of the workshop. Nevertheless, it could also be considered a strength because the variability better reflects how the workshop would be implemented in a real-world setting across various countries. There is a need to evaluate the effectiveness of interventions and activities in reallife settings that can be extended and implemented in a variety of locations and countries. To date, the effect of cooking on children's behavior has been examined as part of multicomponent nutrition education interventions and has been studied with experimental designs and randomized trials. For activities and events that will take place naturally in realworld settings like these workshops, it is difficult to implement a reference standard of randomized controlled trials. Therefore, natural experiments or field studies evaluating the effectiveness of real-world changes or events can provide an alternative study design.

\section{IMPLICATIONS FOR RESEARCH AND PRACTICE}

This study showed the positive effect of a cooking workshop on children's liking across a selection of countries worldwide. Future studies measuring both pre- and post-workshop intake as well as absolute grams are needed to determine the effect of cooking workshops on fruit and vegetable intake. It remains important to evaluate the long-term effectiveness of interventions and activities in reallife settings that can be extended and implemented in a variety of locations and countries. Methods including study protocols, questionnaires, and data analyses need to be further developed to capture the effects of these events fully. Adapting children's food environment is increasingly seen as fundamental to helping children develop healthy eating habits. Improving the attractiveness of healthier options through visual appeal, modeling, and participation in meal preparation are promising strategies to support healthy eating patterns in children.

\section{ACKNOWLEDGMENTS}

This study was funded by Nestec Ltd (Vevey, Switzerland).

\section{REFERENCES}

1. Evans CE, Christian MS, Cleghorn CL, Greenwood DC, Cade JE. Systematic review and meta-analysis of schoolbased interventions to improve daily fruit and vegetable intake in children aged 5 to 12 y. Am J Clin Nutr. 2012;96: 889-901.

2. Jaime PC, Lock K. Do school based food and nutrition policies improve diet and reduce obesity? Prev Med. 2009;48:45-53.

3. Peters J, Parletta N, Lynch J, Campbell K. A comparison of parental views of their pre-school children's "healthy" versus "unhealthy" diets: a qualitative study. Appetite. 2014;76:129-136.

4. Orrell-Valente JK, Hill LG, Brechwald WA, Dodge KA, Pettit GS, Bates JE. "Just three more bites": an observational analysis of parents' socialization of children's eating at mealtime. Appetite. 2007;48:37-45.

5. Russell CG, Worsley A, Campbell KJ. Strategies used by parents to influence their children's food preferences. Appetite. 2015;90:123-130.

6. DeCosta P, Moller P, Frost MB, Olsen A. Changing children's eating behaviourA review of experimental research. Appetite. 2017;113:327-357.

7. Hersch D, Perdue L, Ambroz T, Boucher JL. The impact of cooking classes on food-related preferences, attitudes, and behaviors of school-aged children: a systematic review of the 
evidence, 2003-2014. Prev Chronic Dis. 2014;11:140267.

8. Dudley DA, Cotton WG, Peralta LR. Teaching approaches and strategies that promote healthy eating in primary school children: a systematic review and meta-analysis. Int J Behav Nutr Phys Act. 2015;12:28.

9. Zeinstra GG, Koelen MA, Kok FJ, van der Laan N, de Graaf C. Parental childfeeding strategies in relation to Dutch children's fruit and vegetable intake. Public Health Nutr. 2010;13:787-796.

10. Rohlfs Dominguez P, Gamiz F, Gil M, et al. Providing choice increases children's vegetable intake. Food Qual Prefer. 2013;30:108-113.

11. Heath P, Houston-Price C, Kennedy OB. Increasing food familiarity without the tears: a role for visual exposure? Appetite. 2011;57:832-838.

12. Aldridge V, Dovey TM, Halford JCG. The role of familiarity in dietary development. Dev Rev. 2009;29:32-44.

13. Dougherty K, Silver C. Chef-nutritionist teams spark enjoyment and learning in cooking education series for 8- to 12 year-olds. J Nutr Educ Behav. 2007;39: 237-238.

14. Heim S, Stang J, Ireland M. A garden pilot project enhances fruit and vegetable consumption among children. J Am Diet Assoc. 2009;109:1220-1226.

15. Franke N, Schreier M, Kaiser U. The "I designed it myself" effect in mass customization. Manage Sci. 2010;56:125140.

16. Norton MI, Mochon D, Ariely D. The IKEA effect: when labor leads to love. J Consum Psychol. 2012;22:453-460.

17. Dohle S, Rall S, Siegrist M. I cooked it myself: preparing food increases liking and consumption. Food Qual Prefer. 2014;33:14-16.

18. van der Horst K. Overcoming picky eating. Eating enjoyment as a central aspect of children's eating behaviors. Appetite. 2012;58:567-574.

19. Galloway AT, Fiorito LM, Francis LA, Birch LL. "Finish your soup": counterproductive effects of pressuring children to eat on intake and affect. Appetite. 2006;46:318-323.

20. van der Horst K, Ferrage A, Rytz A. Involving children in meal preparation: effects on food intake. Appetite. 2014;79:18-24.

21. Allirot X, da Quinta N, Chokupermal $\mathrm{K}$, Urdaneta E. Involving children in cooking activities: a potential strategy for directing food choices toward novel foods containing vegetables. Appetite. 2016;103:275-285.

22. Chu YL, Storey KE, Veugelers PJ. Involvement in meal preparation at home is associated with better diet quality among Canadian children. J Nutr Educ Behav. 2014;46:304-308.

23. Leech RM, McNaughton SA, Crawford DA, Campbell KJ, Pearson N, Timperio A. Family food involvement and frequency of family dinner meals among Australian children aged 10-12 years: cross-sectional and longitudinal associations with dietary patterns. Appetite. 2014;75:64-70.

24. Larson NI, Story M, Eisenberg ME, Neumark-Sztainer D. Food preparation and purchasing roles among adolescents: associations with sociodemographic characteristics and diet quality. J Am Diet Assoc. 2006;106:211-218.

25. Yee AZ, Lwin MO, Ho SS. The influence of parental practices on child promotive and preventive food consumption behaviors: a systematic review and meta-analysis. Int J Behav Nutr Phys Act. 2017;14:47.

26. Ong JX, Ullah S, Magarey A, Miller J, Leslie E. Relationship between the home environment and fruit and vegetable consumption in children aged 612 years: a systematic review. Public Health Nutr. 2017;20:464-480.

27. Cullen KW, Baranowski T, Owens E, Marsh T, Rittenberry L, de Moor C. Availability, accessibility, and preferences for fruit, $100 \%$ fruit juice, and vegetables influence children's dietary behavior. Health Educ Behav. 2003;30:615-626.

28. van Kleef E, Vrijhof M, Polet IA, Vingerhoeds $\mathrm{MH}$, de Wijk RA. Nudging children towards whole wheat bread: a field experiment on the influence of fun bread roll shape on breakfast consumption. BMC Public Health. 2014;14:906.

29. Jansen E, Mulkens S, Jansen A. How to promote fruit consumption in children: visual appeal versus restriction. Appetite. 2010;54:599-602.
30. Nestlé. Nestlé Healthy Kids Programme. https://www.nestle.com/csv/globalinitiatives/healthier-kids. Accessed February 25, 2019

31. Commission cantonale d'éthique de la recherche sur l'être humain (Cantonal Commission on Ethics in Human Research) [in French]. http://www. cer-vd.ch/soumission/premiers-pas. html\#c752. Accessed March 18, 2019.

32. Bradley MM, Lang PJ. Measuring emotion: the Self-Assessment Manikin and the Semantic Differential. J Behav Ther Exp Psychiatry. 1994;25:49-59.

33. Bennett $\mathrm{C}$, Blissett J. Measuring hunger and satiety in primary school children: validation of a new picture rating scale. Appetite. 2014;78:40-48.

34. Marty L, Chambaron S, Nicklaus S, Monnery-Patris S. Learned pleasure from eating: an opportunity to promote healthy eating in children? Appetite. 2018;120:265-274.

35. Olsen A, Ritz C, Kramer L, Moller P. Serving styles of raw snack vegetables: what do children want? Appetite. 2012; 59:556-562.

36. Chung LMY, Fong SSM. Appearance alteration of fruits and vegetables to increase their appeal to and consumption by school-age children: a pilot study. Health Psychol Open. 2018;5: 2055102918802679.

37. Hass J, Hartmann M. What determines the fruit and vegetables intake of primary school children? An analysis of personal and social determinants. Appetite. 2018;120:82-91.

38. Draxten M, Fulkerson JA, Friend S, Flattum CF, Schow R. Parental role modeling of fruits and vegetables at meals and snacks is associated with children's adequate consumption. Appetite. 2014;78:1-7.

39. Ahern SM, Caton SJ, Blundell-Birtill P, Hetherington MM. The effects of repeated exposure and variety on vegetable intake in pre-school children. Appetite. 2019;132:37-43.

40. Lakkakula A, Geaghan J, Zanovec M, Pierce S, Tuuri G. Repeated taste exposure increases liking for vegetables by low-income elementary school children. Appetite. 2010;55:226231. 
925.e1 van der Horst et al

CONFLICT OF INTEREST

K.v.d.H. and K.C.M. were employed by Nestec Ltd during the time of the
Volume 51, Number 8, 2019

study. A.P.P. is currently employed with Nestec Ltd. X.A. declares that he has no relevant conflicts of interest. 